\title{
Anti-inflammatory effect of galectin-1 in a murine model of atopic dermatitis
}

\author{
Mab Pereira Corrêa ${ }^{1} \cdot$ Frans Eberth Costa Andrade ${ }^{2} \cdot$ Alexandre Dantas Gimenes $^{2}$ • \\ Cristiane Damas Gil ${ }^{1,2}$
}

Received: 24 January 2017 /Revised: 12 May 2017 / Accepted: 29 May 2017 /Published online: 29 June 2017

(C) Springer-Verlag GmbH Germany 2017

\begin{abstract}
Atopic dermatitis $(\mathrm{AD})$ is caused by both dysregulated immune responses and an impaired skin barrier. Although betagalactoside-binding protein galectin-1 (Gal-1) has immunomodulatory effects in several inflammatory disorders, therapeutic strategies based on its anti-inflammatory properties have not been explored in $\mathrm{AD}$. Thus, we evaluate pharmacological treatment with Gal-1 in the progression of an ovalbumin (OVA)induced AD-like skin lesions. The skin of OVA-immunized male BALB/c mice was challenged with drops containing OVA on days 11, 14-18 and 21-24. Additionally, in the last week, a subset of animals was treated intraperitoneally with recombinant Gal-1 (rGal-1) or dexamethasone (Dex). Treatment with rGal-1 decreased the clinical signs of dermatitis in BALB/c mice and diminished local eotaxin and IFN- $\gamma$ levels. The treatment also suppressed the infiltration of eosinophils and mast cells, which was verified by reduced expression of mouse mast cell protease 6 (mMCP6) and eosinophil peroxidase (EPX). These localized effects are associated with extracellular signal-regulated kinase (ERK) activation and downregulation of endogenous Gal-1. The inhibition of disease progression induced by rGal-1 was also correlated with reduced plasma IL-17 levels. Our results demonstrate that rGal1 is an effective treatment for allergic skin inflammation in $\mathrm{AD}$ and may impact the development of novel strategies for skin inflammatory diseases.
\end{abstract}

Cristiane Damas Gil

cristiane.gil@unifesp.br

1 Post-Graduation in Biosciences, UNESP - São Paulo State University, São José do Rio Preto 15054-000, Brazil

2 Department of Morphology and Genetics, UNIFESP - Federal University of São Paulo, São Paulo 04023-900, Brazil

\section{Key messages}

- Pharmacological treatment with rGal-1 reduces clinical signs of atopic dermatitis.

- Systemic treatment with rGal-1 inhibits eosinophil and mast cell influx in the skin of AD animals.

- rGal-1 reduced local eotaxin levels and systemic IL-17 levels.

- The inhibition of disease progression induced by rGal-1 was correlated with upregulation of phosphorylated ERK.

Keywords Galectin-1 · Skin inflammation · Ovalbumin · Eosinophil $\cdot$ Mast cell $\cdot$ ERK

\section{Introduction}

Atopic dermatitis (AD), also known atopic eczema, represents the most common chronic inflammatory skin disease, characterized by itchy, red, swollen and cracked skin $[1,2]$. AD etiology is multifactorial, and there is evidence that genetic predisposition and family history for atopic diseases, such as bronchitis, asthma and rhinitis, influence $\mathrm{AD}$ development.

The pathogenesis of $\mathrm{AD}$ is attributed to an imbalance in the adaptive immune system, including dysfunction in $\mathrm{T}$ helper (Th) cells and increased IgE production [1-3]. Cytokines and chemokines, such as interleukin (IL)-4, IL-5, IL-13, eotaxins, C-C motif chemokine ligand (CCL) 17, CCL18 and CCL22, produced by Th2 cells and dendritic cells stimulate the infiltration of mast cells and eosinophils into the skin. Th2 and Th17 lymphocytes predominate in patients with $\mathrm{AD}$, but Th1 cells also contribute to the pathogenesis. Cytokines produced by Th2 and Th17 cells (IL-4/IL-13 and IL-17/IL-22, respectively) inhibit terminal differentiation of the epidermis and contribute to breakdown of the epithelial barrier in patients with $\mathrm{AD}$. 
There are a number of treatments for $\mathrm{AD}$ which include emollients, dexamethasone, topical glucocorticoids, calcineurin inhibitors, phototherapies and immunosuppressant such as cyclosporine A, which are effective in reducing inflammation but cause adverse effects $[1,2]$. Thus, the discovery of new pharmacological agents that have high efficacy in controlling the inflammatory response with fewer side effects is critical.

Galectin-1 (Gal-1) is a $14.5-\mathrm{kDa} \beta$-galactoside-binding mammalian lectin with anti-inflammatory properties demonstrated in vitro and in vivo experimental models. In vitro, incubation with recombinant Gal-1 (rGal-1) inhibits the migration of human neutrophils [4] and lymphocytes [5] through endothelial cells following IL- 8 or TNF- $\alpha$ stimulation, respectively. In vivo, rGal-1 administration is associated with inhibition of neutrophil extravasation into the peritoneal cavity after $4 \mathrm{~h}$ of carrageenan- [6], zymosan- [7], or IL- $1 \beta$-induced peritonitis in rodents [4]. The anti-migratory effect of Gal-1 is also associated with the modulation of L-selectin and $\beta 2$-integrin expression on the surface of leukocytes [5, 7, 8], but not E-selectin, ICAM1 and VCAM-1 on endothelial cells [5]. In a mouse model of ovalbumin-induced allergic conjunctivitis, we have recently shown that rGal-1 inhibits IL-4, IL-13 and eotaxin levels in lymph nodes and is associated with reduced clinical signs of disease and decreased plasma IgE anti-ovalbumin levels [9]. However, the mechanisms by which Gal-1 modulates cellular responses in allergic inflammatory processes are not still completely determined, especially in the skin.

In addition to these immunomodulatory roles, the signal transduction events that lead to Gal-1 induced neutrophil and endothelial cell migration and T-cell death include the activation of mitogen-activated protein kinases (MAPKs) $[10,11]$. MAPKs, well-conserved signaling pathways, include three subtypes: c-jun N-terminal kinases (JNK), extracellular signal-regulated kinases (ERK) and p38 proteins, which are crucial to induce the expression of multiple genes that together regulate the immune responses [12]. This kinase family has been implicated in allergic responses, particularly in asthma, contributing to leukocyte recruitment, mast cell activation, pro-inflammatory cytokine production and differentiation of Th2 and Th17 lymphocytes [13, 14].

Given the known anti-inflammatory effects of Gal-1 and the current limitations in the treatment of $\mathrm{AD}$, we evaluated the mechanism of action of this protein in an experimental model of ovalbumin-induced $\mathrm{AD}$ in mice.

\section{Material and methods}

\section{Animals}

Male BALB/c mice, weighing 20-25 g, were randomly distributed into five groups ( $n=6$ animals/group). The animals were housed in a 12-h light-dark cycle and were allowed food and water ad libitum. All animal procedures were approved by the Ethics Committee in Animal Experimentation of the Federal University of São Paulo-UNIFESP (CEP 1906060115/2015).

\section{Allergic dermatitis model and treatment protocols}

To develop a mouse model of $\mathrm{AD}$ using skin sensitization, $\mathrm{BALB} / \mathrm{c}$ mice were immunized on days 0 and 7 with a subcutaneous injection of $5 \mu \mathrm{g}$ of ovalbumin (OVA; grade V; SigmaAldrich, MO, USA) and $10 \mathrm{mg} / \mathrm{mL}$ of aluminium hydroxide adjuvant diluted in $200 \mu \mathrm{L}$ of sterile saline according to previous studies $[9,15]$ with modifications. On day 11 , animals were shaved and the hair removed from the whole back. The skin of mice was challenged with drops containing $250 \mu \mathrm{g}$ of OVA diluted in $50 \mu \mathrm{L}$ of JOHNSON'S $®$ baby oil on days 11,14 18 and 21-24. In the last week (days 21-24), mice were treated with recombinant Gal-1 protein (rGal-1; Peprotech EC Ltd., London, UK) $3 \mu \mathrm{g} / \mathrm{animal}$, or dexamethasone (Dex) (1 mg/ $\mathrm{kg}$, Sigma-Aldrich) intraperitoneal, diluted in $0.1 \mathrm{~mL}$ of sterile saline 15 min prior OVA challenge. rGal-1 and Dex doses were scaled up from the anti-inflammatory doses described previously $[6,9]$. Control groups were constituted by Naïve (mice were not subjected to any procedures related to the AD model) and Sham (mice that received sterile saline and JOHNSON'S® baby oil alone). Twenty-four hours after the last OVA challenge, mice were anesthetized with ketamine $(100 \mathrm{mg} / \mathrm{kg})$ and xylazine $(20 \mathrm{mg} / \mathrm{kg})$ followed by cardiac puncture using a syringe with $3.8 \%$ sodium citrate to obtain blood. Animals were then euthanized and the skin collected.

\section{Blood leukocyte quantification}

Aliquots of blood $(10 \mu \mathrm{L})$ were diluted $1 / 20$ in Turk's solution ( $0.1 \%$ crystal violet diluted in $3 \%$ acetic acid), and differential counting was obtained with a Neubauer chamber using a $40 \times$ objective on a light microscope. For this study, blood cells were distinguished as lymphocytes, neutrophils, monocytes and eosinophils. Data were reported as the mean \pm SEM of the number of cells $\times 10^{5} / \mathrm{mL}$.

\section{Analysis of IgE anti-ovalbumin and Gal-1 levels}

Blood from various experimental conditions was centrifuged at $600 \times \mathrm{g}$ for $10 \mathrm{~min}$ to collect the plasma and determine the IgE anti-OVA levels by ELISA. The concentration of IgE antiOVA was measured using a commercially available mouse IgE anti-OVA immunoassay kit (Cayman Chemical Co., MI, USA) in accordance with the manufacturer's instructions. Gal-1 levels in skin homogenates were determined using a commercially available kit (RayBiotech, GA, USA), in accordance with the manufacturer's guidelines. All experiments were conducted in duplicate, and the data expressed as the mean \pm SEM protein $(\mathrm{ng} / \mathrm{mL})$. 


\section{Skin thickness, histopathology and quantification of inflammatory cells}

Skins were fixed in $4 \%$ paraformaldehyde for $24 \mathrm{~h}$, washed in tap water, dehydrated in a decreasing ethanol series and embedded in paraffin. Sections of $3 \mu \mathrm{m}$ were obtained in a Leica RM2155 microtome (Leica Microsystems, Nussloch, Germany) and subsequently stained with haematoxylin-eosin or toluidine blue $0.5 \%$ for histopathology and inflammatory cell quantification. Eosinophils, neutrophils and mast cells were quantified using a $40 \times$ objective on an Axio Scope A1 Zeiss microscope (Carls Zeiss, Jena, Germany). Three semi-serial sections of skin were analysed per animal, and the area was determined using AxioVision software (Carl Zeiss). Values are expressed as the mean \pm SEM cells per square millimeter.

A $10 \times$ objective was used to measure skin thickness, with lines perpendicular to the keratin layer of epidermis up to the hypodermis. For each animal, three measurements of the epidermis + dermis were taken at random intervals using AxioVision software (Carl Zeiss). Values are shown as mean \pm SEM of the thickness (mm).

\section{Immunohistochemistry}

Endogenous Gal-1 staining was performed in 3- $\mu \mathrm{m}$ sections of paraffin-embedded skin. After an antigen retrieval step using citrate buffer $\mathrm{pH}$ 6.0, the endogenous peroxide activity was blocked and the sections were incubated overnight at $4{ }^{\circ} \mathrm{C}$ with a rabbit polyclonal anti-Gal-1 antibody (Santa Cruz Biotechnology, CA, USA), diluted 1:200 in PBS 1\% BSA. After washing, sections were incubated with a secondary biotinylated antibody (LAB-SA Detection kit, Invitrogen, Paisley, UK). Positive staining was detected using a peroxidaseconjugated streptavidin complex, and colour was developed using DAB substrate (Invitrogen). The sections were counterstained with haematoxylin.

Densitometry analysis of Gal-1 immunostaining was performed in the epidermis and dermis ( $n=6$ animals/group). The values were obtained as arbitrary units (a.u.) between 0 and 255 using AxioVision software on an Axioskop 2 mot plus Zeiss microscope (Carl Zeiss, Jena, Germany). The data are expressed as the mean \pm SEM of a. $\mathrm{u}$.

\section{Western blot analysis}

Skins were sonicated in a $50 \mathrm{mM}$ Tris- $\mathrm{HCl}, 150 \mathrm{mM} \mathrm{NaCl}$ and $1 \%$ Triton-X, pH 7.4 buffer containing a complete protease inhibitor cocktail and PhosSTOP tablets (Roche Applied Science, Mannheim, Germany). Subsequently, samples were centrifuged at $10,000 \times g$ for $20 \mathrm{~min}$ at $4{ }^{\circ} \mathrm{C}$ to obtain organ supernatants. Protein levels were determined by the Bradford assay and normalized prior to boiling in Laemmli buffer (BioRad Laboratories, USA). Pooled protein extracts $(30 \mu \mathrm{g}$ per lane) of skin ( $n=3$ animals per group) from indicated experimental conditions were loaded onto a $12 \%$ sodium dodecyl sulphate-polyacrylamide gel for electrophoresis together with appropriate molecular weight markers (Bio-Rad Life Science, USA) and transferred to ECL Hybond nitrocellulose membranes. Reversible protein staining of the membranes with $0.1 \%$ Ponceau-S in $5 \%$ acetic acid (Santa Cruz Biotechnology) was used to verify protein transfer. Membranes were incubated for $15 \mathrm{~min}$ in 5\% BSA in Trisbuffered saline (TBS) prior to incubation with antibodies. Primary antibodies used herein, rabbit polyclonal antibody anti-ERK1/2 (1:5000); mouse monoclonal anti-phosphorylated (p)-ERK $1 \frac{1}{2}$ (1:2000) (Cell Signaling, Danvers, USA); goat polyclonal anti-mouse mast cell protease 6 (mMCP6; 1:5000) (R\&D Systems, Minneapolis, USA); anti-eosinophil peroxidase (EPX; 1:200) (Santa Cruz Biotechnology); rabbit polyclonal antiintegrin $\alpha \mathrm{M}(\mathrm{CD} 11 \mathrm{~b} ; 1: 200)$ (Santa Cruz Biotechnology); anti-GAPDH (1:5000) (Sigma-Aldrich); and rat monoclonal anti-CD4 (1:500) (Rheabiotech-Imuny, Campinas, Brazil), all diluted in TBS with $0.1 \%$ Tween 20 . Post primary antibody incubation, membranes were washed for 15 min with TBS and subsequently incubated for $60 \mathrm{~min}$ at room temperature with the appropriate secondary antibodies. Secondary antibodies were peroxidase-conjugated goat anti-rabbit IgG, goat anti-rat, rabbit anti-goat (1:2000) (Thermo Fisher Scientific Inc., MI, USA) or goat anti-mouse (1:2000) (Millipore Corporation, CA, USA). Finally, membranes were washed for $15 \mathrm{~min}$ with TBS, and immunoreactive proteins were detected (Westar Nova 2.0 chemiluminescent substrate kit; Cyanagen, Bologna, Italy) using a GeneGnome5 chemiluminescence detection system (SynGene, Cambridge, UK). Proteins were imaged and quantified using the software GeneTools (SynGene) to determine the relative expression of indicated proteins (a.u.).

\section{Analysis of cytokines and chemokines}

Multiplex analysis was performed with $25 \mu \mathrm{L}$ of the skin supernatants and plasma using the MILLIPLEX MAP mouse cytokine/chemokine panel (MT17MAG47K-PX25; Millipore Corporation, USA) and MAGPIX® Multiplexing Instrument (Millipore) according to the manufacturer's instructions. Eight analytes were measured: IL-4, IL-10, IL-13, IL-17A, interferon gamma (IFN- $\gamma$ ), tumor necrosis factor alpha (TNF- $\alpha$ ), eotaxin and RANTES (Regulated upon Activation Normal T cell Expressed and presumably secreted). The concentration of analytes was determined by MAGPIX Xponent software (Millipore Corporation, MA, USA), and the results are reported as the mean \pm SEM of analytes $(\mathrm{pg} / \mathrm{mL})$.

\section{Statistical analysis}

The data were analysed using GraphPad Prism 5.0 software. Results were confirmed to follow a normal distribution using 
the Kolmogorov-Smirnov test of normality with DallalWilkinson-Lillie for corrected $P$ value. Data that passed the normality assumption was analysed using ANOVA with the Bonferroni post hoc test. Data that failed the normality assumption were analysed using the nonparametric KruskalWallis followed by the Dunn post-test. Differences were considered statistically significant at a value of $P<0.05$.

\section{Results}

\section{Pharmacological treatment with rGal-1 reduces clinical signs of AD}

Clinical assessment of $\mathrm{AD}$ and skin thickness measurements were performed by macroscopic and histological analysis of mouse skins on the final day of the experimental protocol (day 24). The AD group exhibited skin with erythema, erosion and dryness associated with a marked epidermal hyperplasia and increase in skin thickness compared to the control groups (Naive and Sham) (Fig. 1). Intraperitoneal treatment with rGal-
1 or Dex reduced this $\mathrm{AD}$ clinical response, leaving skins with a similar appearance to control animals. Furthermore, rGal-1 or Dex-treated animals displayed a diminished skin thickness and mild epidermal hyperplasia in relation to the AD group (Fig. 1).

\section{rGal-1 regulates systemic immune responses}

We first assessed the systemic immune response by measuring OVA-specific plasma IgE levels. Epicutaneous serial OVA administration significantly increased the plasma anti-OVA IgE concentration in all AD groups (AD: $231 \pm 28$; rGal-1: $257 \pm 45$; Dex: $205 \pm 28 \mathrm{ng} / \mathrm{mL}$ ) in relation to control groups $(P<0.001)$, supporting the efficacy of our experimental model.

Next, we evaluated the leukocyte recruitment from the blood due to the allergic response induced by epicutaneous challenge with OVA. Blood eosinophilia was exhibited by the ADuntreated and rGal-1 or Dex-treated groups at 24 h (Fig. 2a). Furthermore, $\mathrm{AD}$ induced lymphocytosis and monocytosis that were abrogated by the rGal-1 treatment (Fig. 2c, d) whereas Dex induced only a reduction in the monocyte numbers. No differences were detected in the blood neutrophil numbers (Fig. 2b).
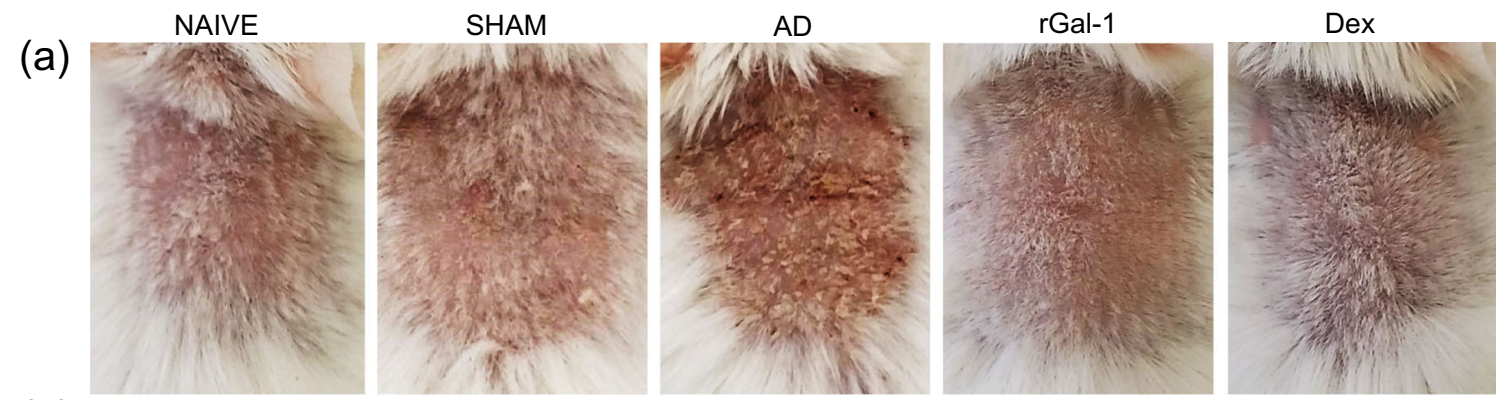

(b)
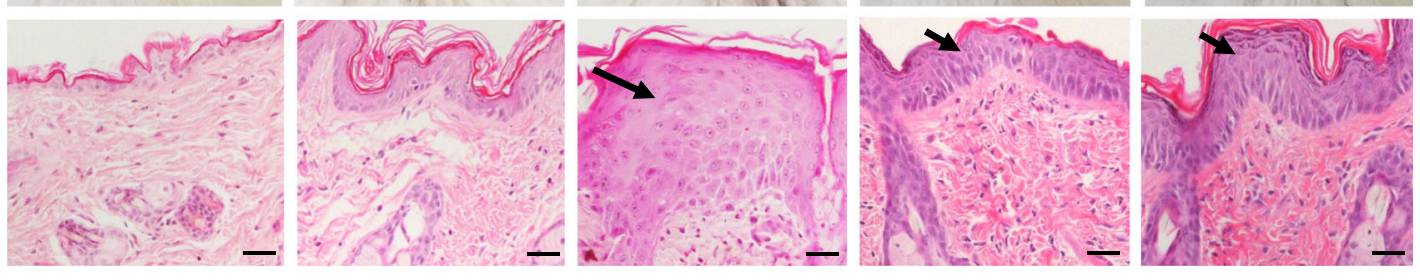

(c)

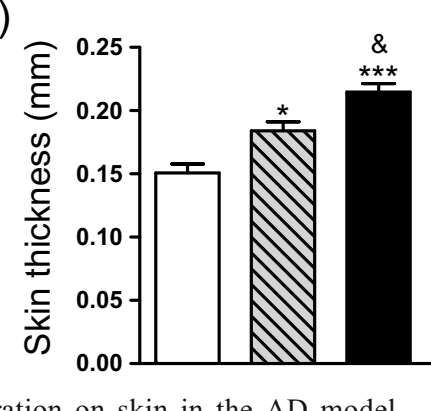

Fig. 1 Effect of rGal-1 administration on skin in the AD model. Macroscopic and histological analyses of skin lesions were taken on the final day of the experiment (day 24). a AD skin exhibited erythema, erosion and dryness whereas rGal-1 and Dex treatments abrogated this effect showing a similar skin appearance as controls (Naïve and Sham). b Histologically, control animals showed normal epidermis with continuous basal layer, 1-2 cell thick spinous layer and thin stratum

corneum. AD results in marked hyperplasia in the epidermis (arrow) with prominent thickened stratum spinosum (arrow). Note mild epidermal hyperplasia (short arrows) after rGal-1 and Dex treatments. Stain: haematoxylin-eosin. Bars: $50 \mu \mathrm{m}$. c Skin thickness. Data represents mean \pm SEM of skin thickness $(\mathrm{mm})(n=6 /$ group $) . * P<0.05$; $* * * P<0.001$ vs Naive; ${ }^{\&} P<0.05 ;{ }^{\& \& \&} P<0.001$ vs Sham; ${ }^{\# \# \#} P<0.001$ vs AD,${ }^{++} P<0.01$ vs rGal-1 (ANOVA, Bonferroni post-test) 
Fig. 2 Effect of rGal-1 on systemic immune responses in the AD model. a-d Quantification of blood eosinophils, neutrophils, lymphocytes and monocytes. Data represent the mean \pm SEM of the number of leukocytes $\times$ $10^{5} / \mathrm{mL}$ in mice under different experimental conditions $(n=6 /$ group). $* P<0.05, * * P<0.01$, $* * * P<0.001$ vs Naive;

${ }^{\&} P<0.05 ;{ }^{\& \&} P<0.01$ vs Sham; ${ }^{\#} P<0.05,{ }^{\# \#} P<0.01$ vs AD (ANOVA, Bonferroni post-test) (a)

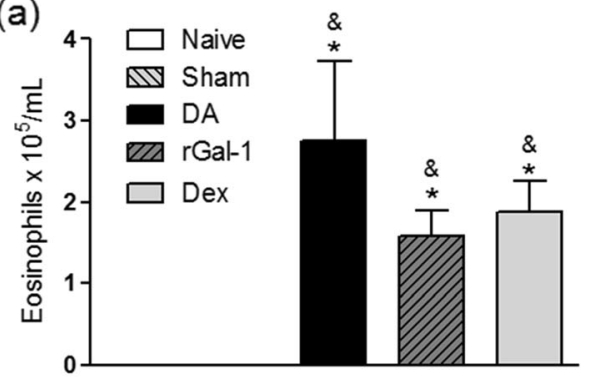

(c)

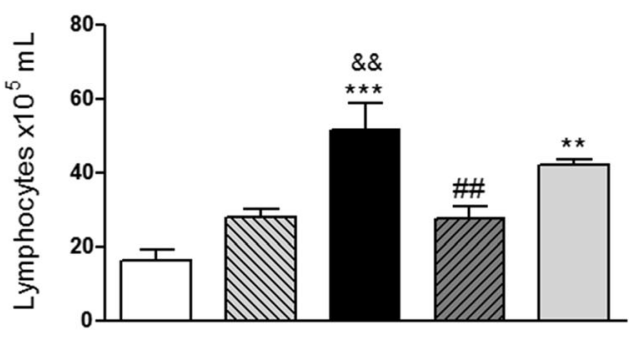

(b)

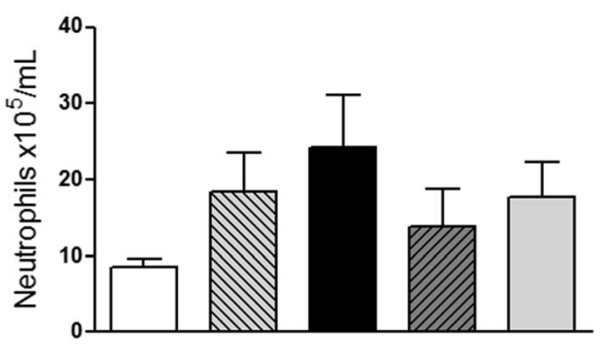

(d)

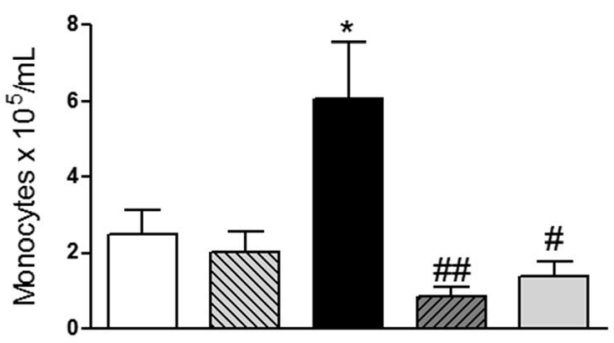

Levels of the chemokines and cytokines belonging to Th1, Th2 and Th17 pathways were analysed by multiplex assay in plasma samples of all experimental groups. Both rGal-1 and Dex treatments markedly reduced plasma IL-17A levels compared to the $\mathrm{AD}$ group (Fig. 3g), while rGal-1 alone diminished the plasma eotaxin concentration (Fig. 3a). However, multiplex immunoassays revealed no significant difference in the plasma levels of RANTES, IL-4, IL-13, IL-10 or IFN- $\gamma$ between the untreated $\mathrm{AD}$ and the Dex- and rGal-1-treated AD groups (Fig. 3b-f).

\section{Effect of rGal-1 on the inflammatory response of the skin and the ERK pathway}

Histopathological analysis of control skins revealed a normal morphology (Fig. 4a, e). In contrast, sensitized and OVAchallenged mice exhibited an inflammatory response characterized by significant infiltration of inflammatory cells in the dermis, particularly eosinophils and degranulated mast cells (Fig. 4b, f). Treatment with rGal-1 and Dex resulted in fewer inflammatory cells in the dermis compared to the untreated AD group (Fig. 4c, d, g, h).

Inflammatory cell counts confirmed the histological observations with significant increase of eosinophils, neutrophils and mast cells in AD group compared to the controls (Fig. 4i). Conversely, rGal-1 and Dex administration produced a significant decrease in eosinophil and neutrophil influx (Fig. 4i).

The presence of inflammatory cells in the mouse skins was also confirmed by immunoblot analysis. T cell influx in $\mathrm{AD}$ groups (treated and untreated) was evidenced by CD4 expression which demonstrated mild increased levels in the rGal-1and Dex-treated groups (Fig. 4j). On the other hand, both treated groups exhibited reduced levels of endogenous adhesion molecule $\mathrm{CD} 11 \mathrm{~b}, \mathrm{EPX}$ and $\mathrm{mMCP} 6$ compared to $\mathrm{AD}$ group which corroborates histological data. In the same way, diminished levels of eotaxin, RANTES and IFN- $\gamma$ were detected in skin homogenates from both treated groups, but only Dex treatment caused a significant reduction compared to AD group (Fig. 5ac). On the other hand, rGal-1 increased TNF- $\alpha$ and IL-10 levels (Fig. 5d, g). IL-4 and IL-13 levels showed no differences between $\mathrm{AD}$ and rGal-1 and Dex-treated groups (Fig. 5e, f).

To understand the downstream systemic molecular signalling pathways involved regulated by $\mathrm{rGal}-1$ treatment in $\mathrm{AD}$, we analysed the phosphorylation levels of ERK by Western blot using pooled extracts of skins ( $n=3$ animals per group). rGal-1 and Dex treatments increased p-ERK levels compared with the nontreated AD group (Fig. 5h), suggesting both treatments function via a similar mechanism.

\section{AD induces increased expression of Gal-1 protein}

To further characterize the Gal-1 expression in mouse skin during the AD inflammatory response, we evaluated Gal-1 protein levels by immunohistochemistry.

Gal-1 expression was detected in both the epidermis and dermis under all experimental conditions, consistent with previous studies $[16,17]$ suggesting that the epithelium is a potential source of this lectin. Twenty-four hours after the last epicutaneous OVA challenge, extracellular matrix of the dermis from the $\mathrm{AD}$ group displayed intense immunostaining for Gal-1 compared with controls (Naïve and Sham) (Fig. 6a). rGal-1- and Dex-treated AD groups produced lower Gal-1 immunostaining in the epidermis and dermis compared with the untreated $\mathrm{AD}$ group. No immunostaining was detected in the negative control section (Fig. 6b). The histologic findings were supported by densitometry analyses of Gal-1 expression in the epidermis and dermis (Fig. 6c, d). Similarly, ELISA data 
Fig. 3 Effect of rGal-1 on the chemokine/cytokine systemic release. a-g Multiplex immunoassay of eotaxin (a), RANTES (b), IL-4 (c), IL-13 (d), IL-10 (e), IFN- $\gamma$ (f) and IL-17A (g) in plasma. Values are expressed as the mean \pm SEM of the chemokines/cytokines (pg/ $\mathrm{mL} ; n=6$ /group). ${ }^{\#} P<0.05$ vs AD (Kruskal-Wallis, Dunn posttest)

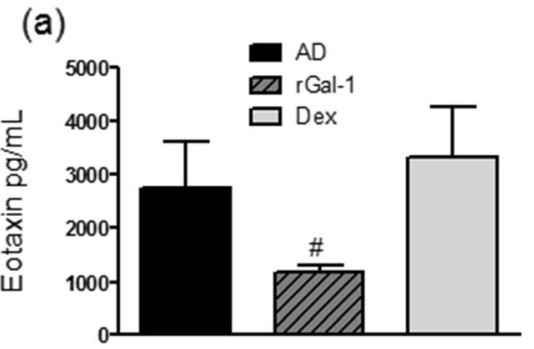

(c)

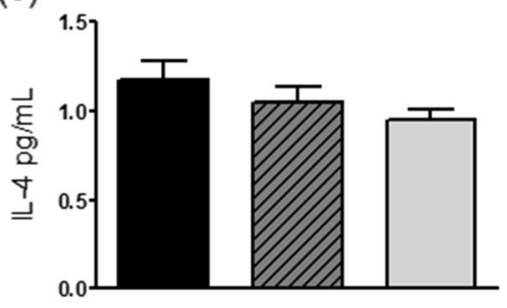

(e)

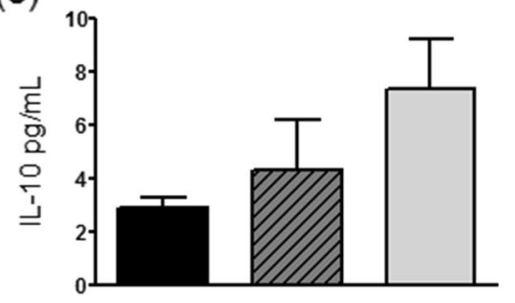

(b)

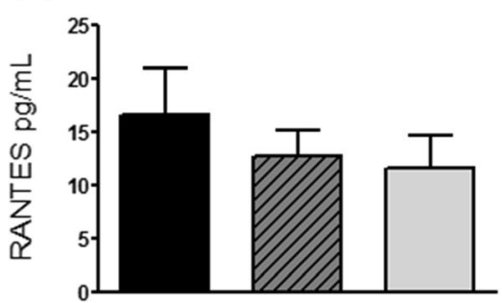

(d)

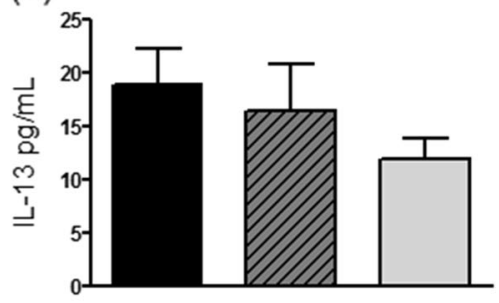

(f)

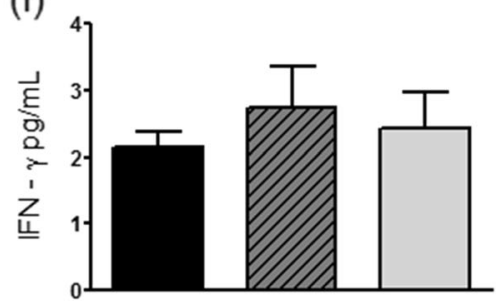

(g)

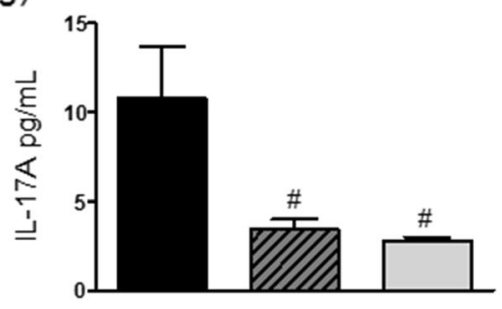

from skin homogenates showed that rGal-1- and Dex-treated samples exhibited decreased endogenous Gal-1 levels compared to AD samples (Fig. 6e).

\section{Discussion}

In this study, we evaluated the effect of pharmacological treatment with recombinant Gal-1 (rGal-1) on OVA-induced AD model in mice. Using macroscopic, histological, biochemical and molecular analyses, we showed that systemic rGal- 1 treatment was as effective as dexamethasone (Dex), in the regulation of the allergic inflammatory response in skin.

The development of $\mathrm{AD}$ was confirmed through the detection of clinical signs such as erythema and dryness, increased skin thickness and plasma anti-OVA IgE levels compared to control animals. Histological analysis revealed epidermal hyperplasia and a marked influx of eosinophils and mast cells in the dermis, which were confirmed by increased EPX and mMCP-6 protein levels in skin

Fig. 4 Histopathology of the skin. a, e Control (Sham and Naive). b, f AD characterized by significant infiltration of eosinophils (arrowheads; inset) and degranulated mast cells (arrows; inset) into the dermis. Pharmacological treatment with $\mathrm{rGal}^{-1}(\mathbf{c}, \mathbf{g})$ and Dex $(\mathbf{d}, \mathbf{h})$ resulted in lesser infiltration of inflammatory cells. Stain: haematoxylin-eosin (a-d) and toluidine blue (e-h). Bars: $50 \mu \mathrm{m}(\mathbf{a}-\mathbf{h})$ and $10 \mu \mathrm{m}$ (insets). i Quantitative analysis of eosinophils, neutrophils and mast cells in the skin. Data represent the mean \pm SEM of the cell number per square millimeter ( $n=6$ animals/group). $* P<0.05$, $* * * P<0.001$ vs control groups (naive and sham); ${ }^{\#} P<0.05,{ }^{\# \#} P<0.01,{ }^{\# \# \#} P<0.001$ vs $\mathrm{AD}$ (ANOVA, Bonferroni post-test). $\mathbf{j}$ Western blot analysis to measure CD11b, CD4, mMCP6 and EPX levels in the pooled extracts of mouse skins ( $n=3$ animals/group) from mice with $\mathrm{AD}$ untreated or treated with rGal-1 or Dex. GAPDH was used as a protein loading control. Immunoreactive bands for proteins were semi-quantified by densitometry and are expressed as arbitrary units (a.u.) of the ratio of CD11b, CD4, mMCP6 or EXP/GAPDH (data represent one illustrative blot from two independent experiments) 


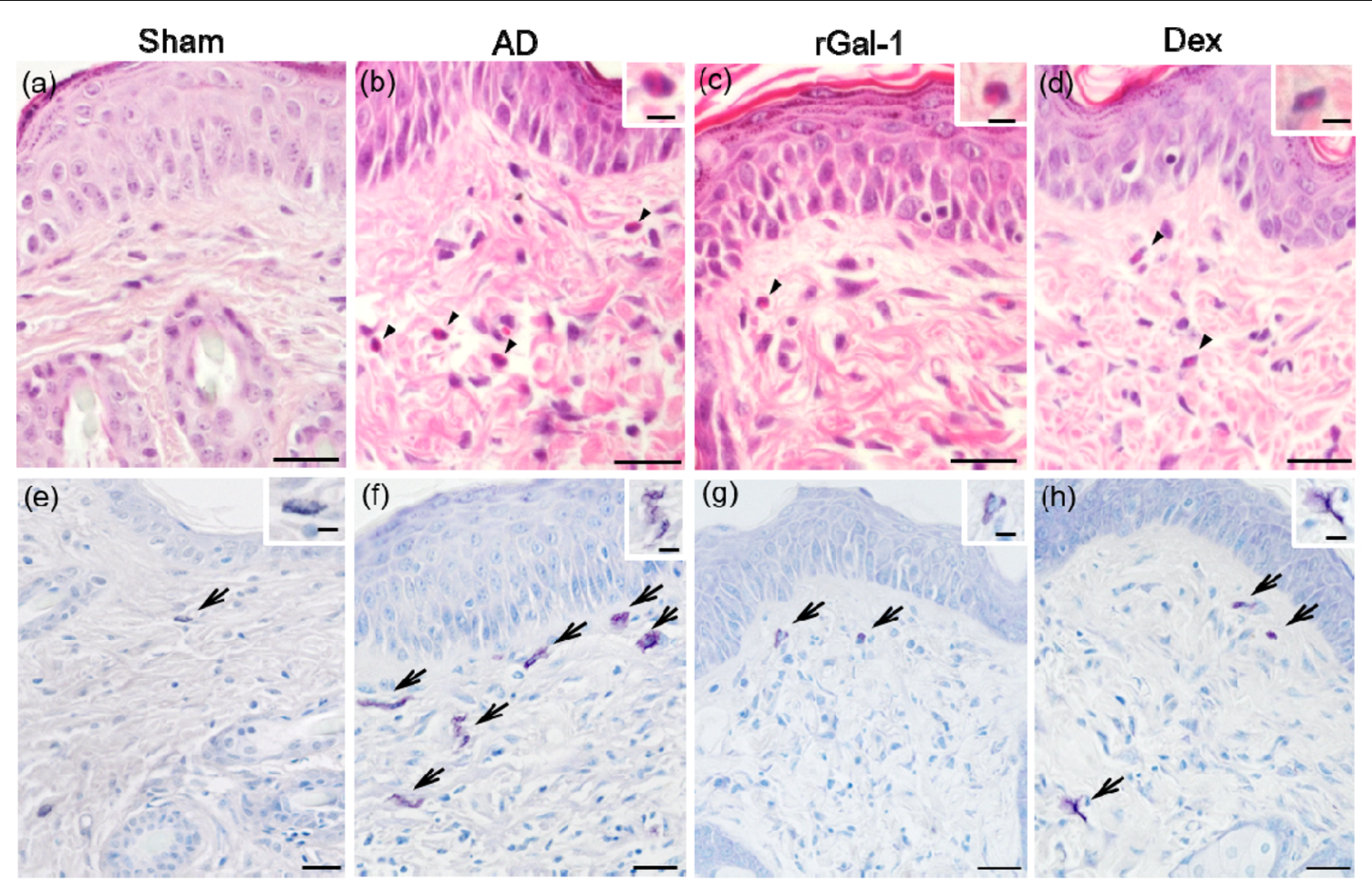

(i)
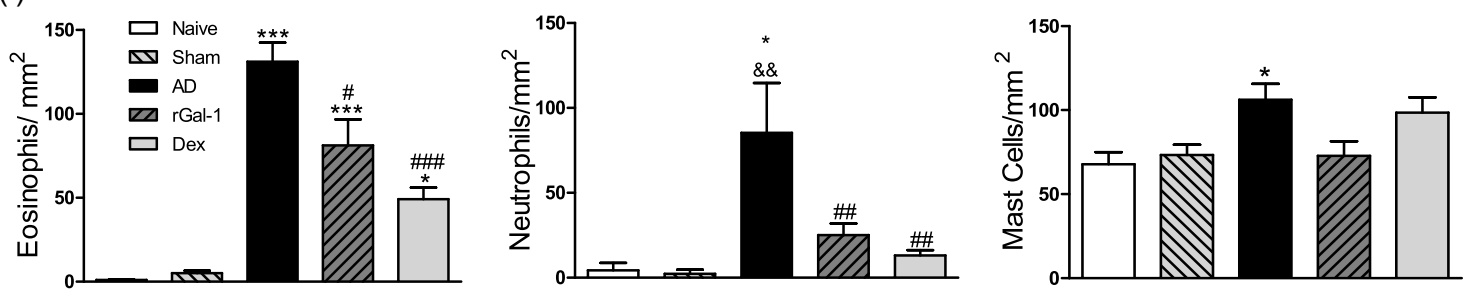

(j)
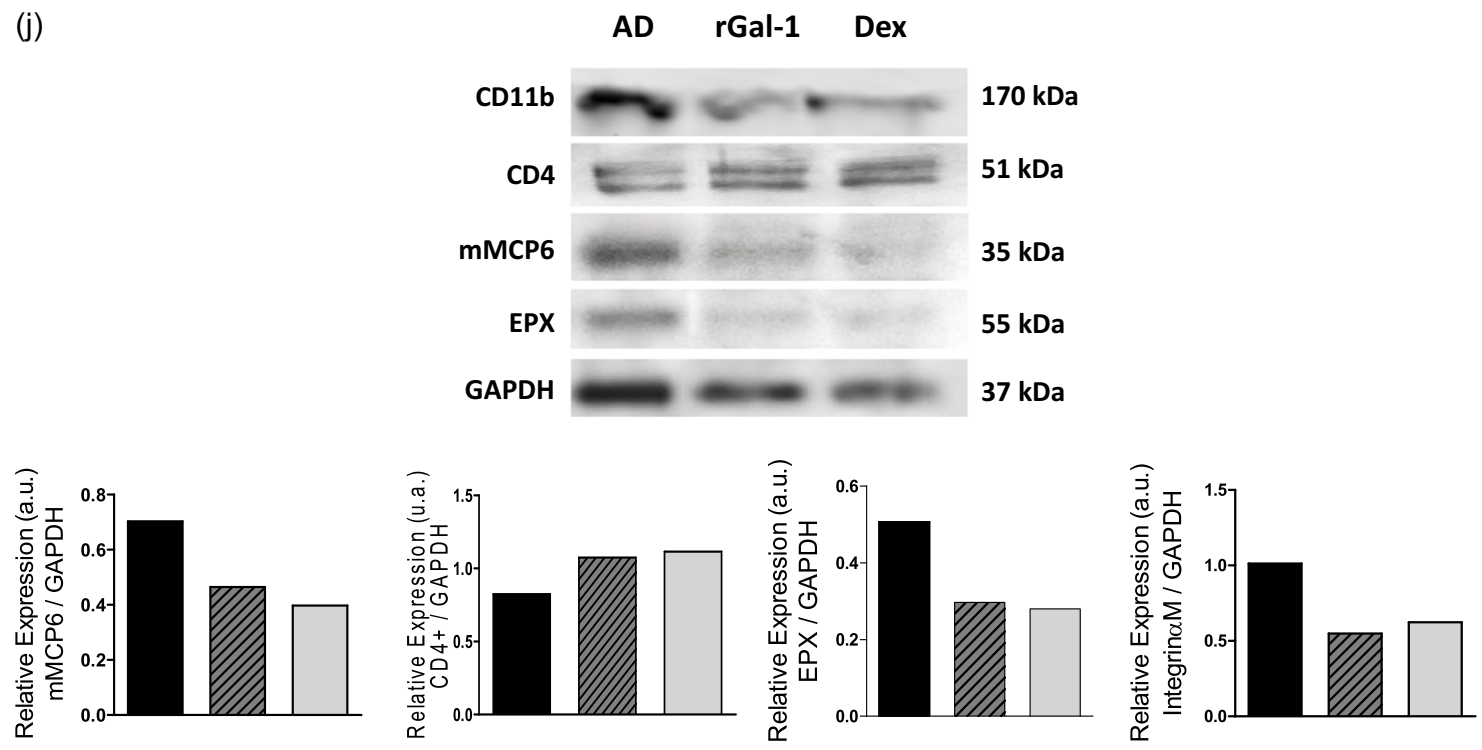

homogenates. Further, blood leukocyte counts revealed eosinophilia, lymphocytosis and monocytosis. These results suggest that external exposure to an allergen can induce localized allergic inflammation in the skin and a systemic sensitization to specific allergen as demonstrated by previous AD models induced by OVA, 2,4dinitrofluorobenzene, 2,4-dinitrochlorobenzene and oxazolone $[15,18,19]$. 
(a)

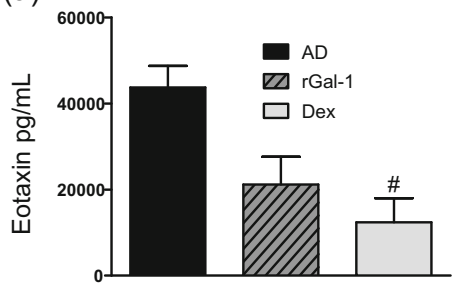

(d)

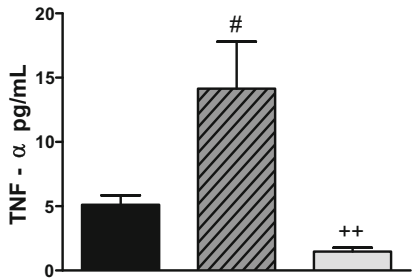

(g)

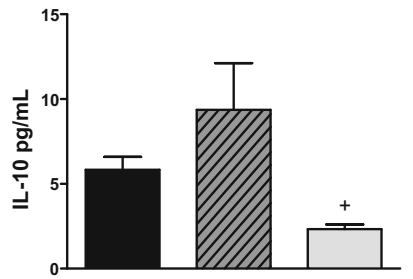

(b)

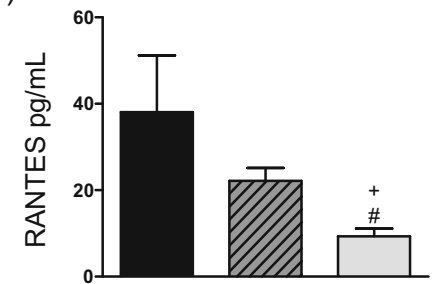

(e)

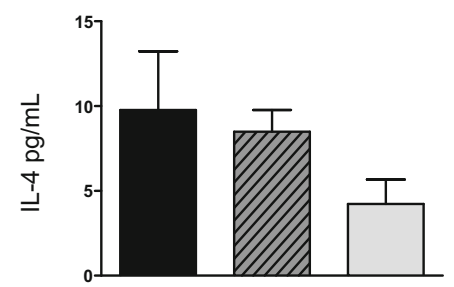

(h)

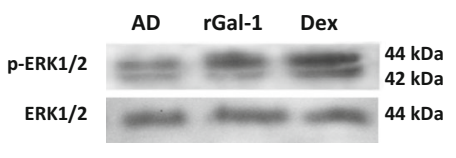

(c)

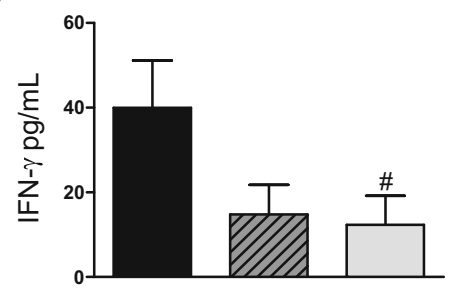

(f)

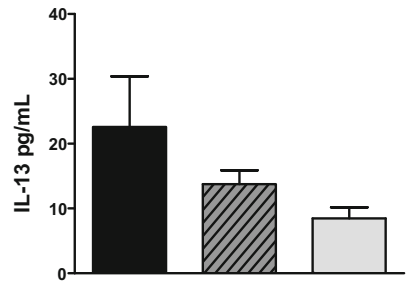

Fig. 5 Effect of rGal-1 administration on local immune response in the AD model. a-c Multiplex immunoassay of skin homogenates to detect eotaxin (a), RANTES (b), IFN- $\gamma(\mathbf{c})$, TNF- $\alpha$ (d), IL-4 (e), IL-13 (f) and IL-10 (g). Values are expressed as the mean \pm SEM of the chemokines/ cytokine (pg/mL; $n=6 /$ group). ${ }^{\#} P<0.05$ vs AD $;{ }^{+} P<0.05,{ }^{++} P<0.05$ vs rGal-1. h Western blot analysis to measure phosphorylated and total ERK levels in the pooled extracts of mouse skins ( $n=3$ animals/group) from mice with $\mathrm{AD}$ untreated or treated with $\mathrm{rGal}-1$ or Dex. Immunoreactive bands for proteins were semi-quantified by densitometry and are expressed as arbitrary units (a.u.) of the ratio of p-ERK/total ERK (data represent one illustrative blot from 2 to 4 independent experiments). ${ }^{\#} P<0.05,{ }^{\# \#} P<0.01$ (Kruskal-Wallis, Dunn post-test)

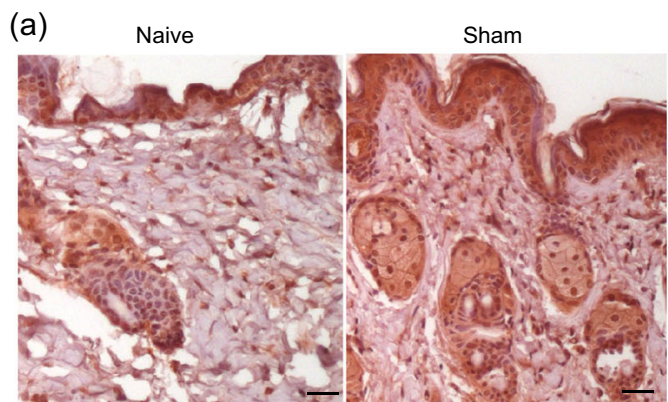

(b) Negative Control

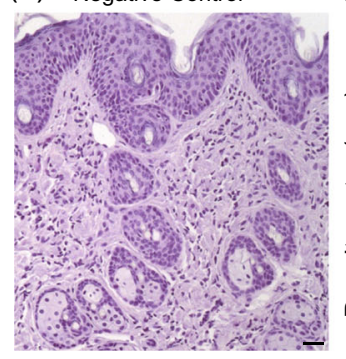

(c)

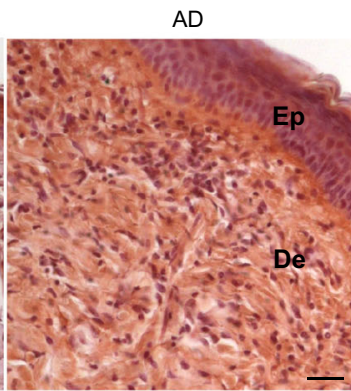

(d)

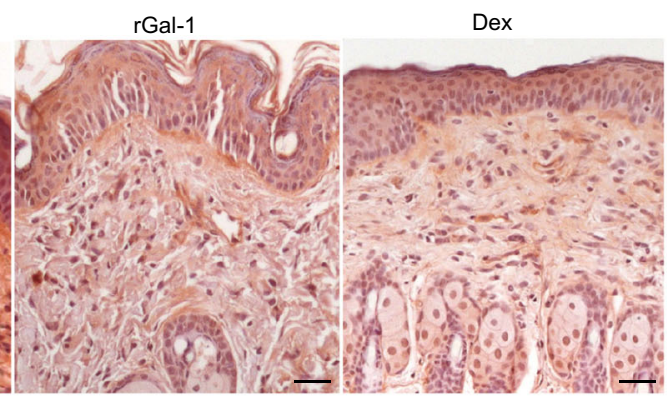

(e)

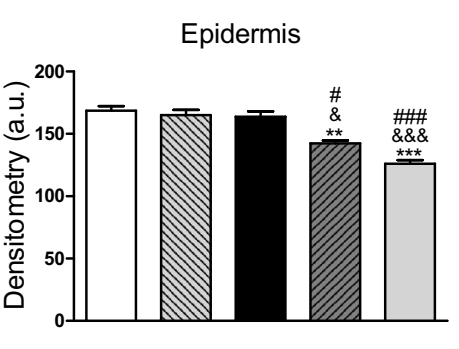

Fig. 6 Expression of Gal-1 in the skin. a Dermis (De) from AD animals showed strong Gal-1 immunostaining compared to the control skins (Naïve and Sham). rGal-1- and Dex-treated groups displayed lower endogenous Gal-1 immunoreactivity in the epidermis (Ep) and dermis (De) compared to the $\mathrm{AD}$ group. $\mathbf{b}$ No staining was detected in the sample used as a negative control. Counterstain: haematoxylin. Bars: $50 \mu \mathrm{m}$. c, d

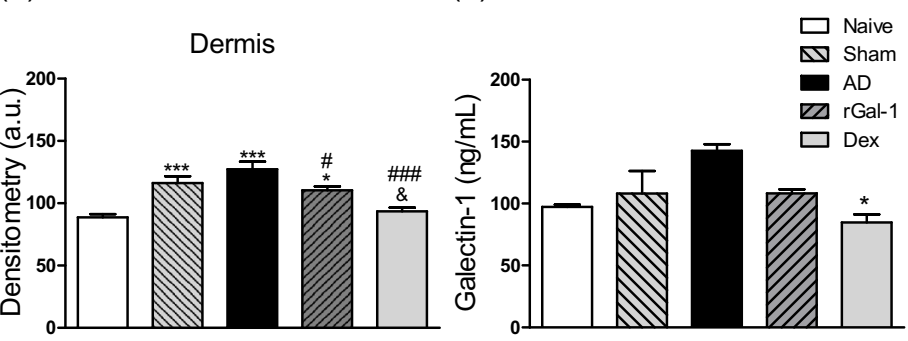

Densitometric analysis of Gal-1 expression in the epidermis and dermis. e Immunoassay for Gal-1 detection in skin homogenates. Data represent mean \pm SEM of Gal-1 expression (a.u.; $n=6$ animals/group). $* P<0.05$; ${ }^{* *} P<0.01, * * * P<0.001$ vs Naive; ${ }^{\circledR} P<0.05 ;{ }^{\& \& \&} P<0.001$ vs Sham; ${ }^{\#} P<0.05$ and ${ }^{\# \# \#} P<0.001$ vs AD (ANOVA, Bonferroni post-test) 
Intraperitoneal administration of rGal-1, as well as Dex, decreased the clinical signs and skin thickness associated with $\mathrm{AD}$, but did not reduce IgE levels $24 \mathrm{~h}$ after the last epicutaneous OVA challenge. Our group has previously shown that systemic rGal-1 and Dex treatments reduced the clinical signs of OVAinduced allergic conjunctivitis in mice; the effect was associated with a significant decrease in the IgE, IL-4 and IL-13 levels $4 \mathrm{~h}$ after the final OVA challenge [9]. Downregulation of $\mathrm{IgE}$ and Th2 cytokine levels by rGal-1 and Dex in the ocular allergy was time dependent once no difference between treated and nontreated groups was detected at $24 \mathrm{~h}$.

Systemic administration of rGal-1 also markedly reduced plasma IL-17A levels compared to the untreated AD group, an important cytokine biomarker for $\mathrm{AD}$ patients [20, 21]. In a previous mouse model, splenocytes isolated from OVAinduced $\mathrm{AD} B A L B / c$ mice had significantly increased levels of IL-4, IL-5, IFN- $\gamma$ and IL-17 after $72 \mathrm{~h}$ of OVA stimulation in vitro, whereas unstimulated cells were not significantly different than control animals [22]. Furthermore, in oxazolone-induced AD IL-17-null mice exhibit reduction of skin edema, eosinophilia, IL-4 and IL-13 production in skin and lymph nodes relative to wild-type animals [19, 23]. The lack of IL-17 expression also ameliorated the skin-barrier dysfunction as evidenced by decreased in transepidermal water loss [19]. The results indicate that the IL-17 gene may play a role in modulating immune dysregulation and affecting skin barrier in $\mathrm{AD}$ mouse models.

It is important to note that Gal-1 plays an essential role in modulating adaptive immune responses by altering the phenotype of $\mathrm{T}$ cells. In this regard, human skin-resident $\mathrm{T}$ cell cultures incubated with a mouse Gal-1 human Ig chimera (Gal-1hFc), and stimulated with phorbol 12-myristate-13acetate (PMA)/ionomycin and brefeldin A, increased IL-4 ${ }^{+}$ and $\mathrm{IL}-10^{+}$lymphocyte populations and decreased IL- $17^{+}$ population [24]. Reinforcing this regulatory aspect of Gal-1 on Th cells, another study found that lymphocytes isolated from Gal-1-null mice with chicken collagen II (CII)-induced arthritis significantly upregulated IL-17 and IL-22 production in vitro after $48 \mathrm{~h}$ of CII stimulation when compared to wildtype cells [25]. Furthermore, $\mathrm{CD}^{+} \mathrm{T}$ cells under Gal-1 treatment increased the secretion of Th2 cytokines and their expansion to $\mathrm{CD}^{+} \mathrm{FOXP}^{+}$Treg cells $[26,27]$. In our AD model, rGal-1 treatment increased plasma and skin levels of IL-10, as well as CD4 expression, in relation to untreated group. Taken together, our data suggest that Gal-1 plays a functional role in the $\mathrm{AD}$ regulation through favouring Treg cell influx and downregulation of IL-17 production.

In addition to regulating Th2/Th17, Gal-1 was able to downregulate Th1 cytokine (IL- $1 \beta$, IFN- $\gamma, \mathrm{TNF}-\alpha$ ) production, as demonstrated in previous studies using experimental models of arthritis [28], colitis [29] and uveitis [30]. The addition of Gal-1 to human monocyte-derived dendritic cell-T cell cocultures from psoriasis patients has also been shown to reduce IFN- $\gamma$ production. This could be reversed by the addition of lactose [31]. Furthermore, it was shown that IFN- $\gamma /$ mast cell axis is involved in the pathology of chronic allergic inflammation of the airways in mice [32]. In the study, IFN- $\gamma$ significantly increased the release of histamine, IL-6 and IL-13 by IgE + specific antigen-stimulated bone marrowderived cultured mast cells in vitro, after 1 or $24 \mathrm{~h}$ of challenge, whereas treatment of the cells with IFN- $\gamma$ alone had no effect. In support of this finding, in our AD model, diminished levels of IFN- $\gamma$ were detected in the skin of rGal-1-treated mice. This reduction correlated with reduced levels of mMCP6 when compared to the untreated AD group, suggesting that the exogenous action of Gal-1 is essential to regulate the activation of mast cells and the initiation of clinical signs of AD.

Another important local effect of rGal-1 discovered in our $\mathrm{AD}$ model is the inhibitory role on eosinophil and neutrophil migration, which was validated by the downregulation of EPX, adhesion molecule CD11b and chemokines eotaxin and RANTES. Similar findings were detected in a OVAinduced allergic conjunctivitis in BALB/c mice, in which pharmacological pretreatment with $\mathrm{rGal}-1$ reduced eotaxin and RANTES levels in the lymph nodes compared with the untreated AC group after $24 \mathrm{~h}$ of last challenge [9]. It was recently shown that the Gal-1-null mice presented increased OVA-induced airway inflammation demonstrated by increased number of eosinophils and lymphocytes in bronchoalveolar fluid and lung and higher airway resistance relative to WT OVA-challenged mice [33].

Our immunohistochemistry studies demonstrated that AD elevated levels of Gal-1 in the dermis in relation to the control groups. On the other hand, exogenous administration of $\mathrm{rGal}-$ 1 downregulated the expression of endogenous Gal-1 protein in the skin, likely as a mechanism of negative feedback. Exposure to the allergen OVA resulted in increased Gal-1 expression in the lungs, eyes and conjunctiva due to the recruitment of Gal-1-expressing inflammatory cells, including eosinophils [9, 33]. Expression of Gal-1 has been demonstrated in the nucleus, cytosol and cell membranes as well as in the extracellular matrix of tissues despite the absence of a secretion signal sequence $[4,7,9]$. Extracellularly, Gal-1 acted as a nonpermissive substrate with respect to processes relevant for eosinophil migration [34]. Similarly, eosinophils exposed to lower concentrations of rGal-1 $(0.1-0.25 \mu \mathrm{M})$ exhibited increased adhesion to recombinant murine to vascular cell adhesion molecule-1 (VCAM-1) in a dose-dependent manner compared with untreated cells under static conditions. However, despite the increased adhesion, eosinophils treated with rGal-1 demonstrated significantly reduced migration toward eotaxin- 1 in in vitro chemotaxis assays [33]. In the same study, in vitro administration of rGal-1 exerted divergent effects on eosinophils that were $\mathrm{N}$-glycan and dose dependent. At low concentrations $(\leq 0.25 \mu \mathrm{M}), \mathrm{rGal}-1$ inhibited ERK1/2 activation and eotaxin-1-induced migration of eosinophils 
while at high concentration $(\geq 1 \mu \mathrm{M})$, induced ERK1/2dependent apoptosis of eosinophils and decreased expression of adhesion molecules CD49d and CCR3. In fact, ERK1/2dependent pathway has also been shown to be critically involved in the regulatory effects of galectin-1 on monocyte activation and T cell viability $[35,36]$.

In line with these previous reports, we herein found in our AD model localized (skin) activation of ERK1/2, plus markedly increased TNF- $\alpha$ levels, following rGal-1 treatment which was not associated with increased inflammatory response. Moreover, recent findings indicate that Gal-1exposed intestinal epithelial cells (IECs) in the presence of proinflammatory cytokines, such as TNF, induced caspasedependent intrinsic pathway of apoptosis [37]. Furthermore, IECs exposed to Gal-1 in the presence of TNF augmented the secretion of epidermal growth fator (EGF), thymic stromal lymphopoietin (TSLP), IL-10 and TGF- $\beta 1$. In the same way, Gal-1 coincubated with IL-13-induced upregulation of IL-10, TGF- $\beta 1$ and IL-25 secretion in IECs. These findings support that Gal-1 can regulate cellular response in an either Th2 or Th1/Th17 proinflammatory microenvironment, as well as, through activation of the ERK pathway in the AD.

Overall, the data presented herein identify Gal-1 as a potential therapeutic agent to regulate $\mathrm{AD}$, as it modulates specific phases of the inflammatory process with clear outcomes under in vivo conditions. We therefore propose that strategies aimed at potentiating the Gal-1 pathway may provide novel pharmacological approaches to the downregulation of the allergic inflammatory response in the skin.

Acknowledgments This study was supported by Fundação de Amparo à Pesquisa do Estado de São Paulo (FAPESP - 2015/09858-3). MP Correa was supported by Conselho Nacional de Desenvolvimento Científico e Tecnológico (CNPq).

Conflict of interest The authors declare no conflict of interest.

\section{References}

1. Leung DY, Guttman-Yassky E (2014) Deciphering the complexities of atopic dermatitis: shifting paradigms in treatment approaches. $\mathrm{J}$ Allergy Clin Immunol 134:769-779

2. Schlapbach C, Simon D (2014) Update on skin allergy. Allergy 69: 1571-1581

3. Dhingra N, Gulati N, Guttman-Yassky E (2013) Mechanisms of contact sensitization offer insights into the role of barrier defects vs. intrinsic immune abnormalities as drivers of atopic dermatitis. J Invest Dermatol 133:2311-2314

4. La M, Cao TV, Cerchiaro G, Chilton K, Hirabayashi J, Kasai K, Oliani SM, Chernajovsky Y, Perretti M (2003) A novel biological activity for galectin-1: inhibition of leukocyte-endothelial cell interactions in experimental inflammation. Am J Pathol 163:1505-1515

5. Norling LV, Sampaio AL, Cooper D, Perretti M (2008) Inhibitory control of endothelial galectin-1 on in vitro and in vivo lymphocyte trafficking. FASEB J 22:682-690
6. Gil CD, Cooper D, Rosignoli G, Perretti M, Oliani SM (2006) Inflammation-induced modulation of cellular galectin-1 and -3 expression in a model of rat peritonitis. Inflamm Res 55:99-107

7. Gil CD, Gullo CE, Oliani SM (2010) Effect of exogenous galectin1 on leukocyte migration: modulation of cytokine levels and adhesion molecules. Int J Clin Exp Pathol 4:74-84

8. Cooper D, Norling LV, Perretti M (2008) Novel insights into the inhibitory effects of galectin-1 on neutrophil recruitment under flow. J Leukoc Biol 83:1459-1466

9. Mello CB, Ramos L, Gimenes AD, Andrade TR, Oliani SM, Gil CD (2015) Immunomodulatory effects of galectin-1 on an IgEmediated allergic conjunctivitis model. Invest Ophthalmol Vis Sci 56:693-704

10. Hsieh SH, Ying NW, Wu MH, Chiang WF, Hsu CL, Wong TY, Jin YT, Hong TM, Chen YL (2008) Galectin-1, a novel ligand of neuropilin-1, activates VEGFR-2 signaling and modulates the migration of vascular endothelial cells. Oncogene 27:3746-3753

11. Auvynet C, Moreno S, Melchy E, Coronado-Martínez I, Montiel JL, Aguilar-Delfin I, Rosenstein Y (2013) Galectin-1 promotes human neutrophil migration. Glycobiology 23:32-42

12. Arthur JS, Ley SC (2013) Mitogen-activated protein kinases in innate immunity. Nat Rev Immunol 13:679-692

13. Pelaia G, Cuda G, Vatrella A, Gallelli L, Caraglia M, Marra M, Abbruzzese A, Caputi M, Maselli R, Costanzo FS et al (2005) Mitogen-activated protein kinases and asthma. J Cell Physiol 202: 642-653

14. Acciani TH, Suzuki T, Trapnell BC, Le Cras TD (2016) Epidermal growth factor receptor signalling regulates granulocytemacrophage colony-stimulating factor production by airway epithelial cells and established allergic airway disease. Clin Exp Allergy 46:317-328

15. Kim H, Kim JR, Kang H, Choi J, Yang H, Lee P, Kim J, Lee KW (2014) 7,8,4'-Trihydroxyisoflavone attenuates DNCB-induced atopic dermatitis-like symptoms in NC/Nga mice. PLoS One 9: e104938

16. Akimoto Y, Hirabayashi J, Kasai K, Hirano H (1995) Expression of the endogenous 14-kDa beta-galactoside-binding lectin galectin in normal human skin. Cell Tissue Res 280:1-10

17. Klíma J, Lacina L, Dvoránková B, Herrmann D, Carnwath JW, Niemann H, Kaltner H, André S, Motlík J, Gabius HJ et al (2009) Differential regulation of galectin expression/reactivity during wound healing in porcine skin and in cultures of epidermal cells with functional impact on migration. Physiol Res 58:873-884

18. Kim HJ, Kim YJ, Kang MJ, Seo JH, Kim HY, Jeong SK, Lee SH, Kim JM, Hong SJ (2012) A novel mouse model of atopic dermatitis with epicutaneous allergen sensitization and the effect of Lactobacillus rhamnosus. Exp Dermatol 21:672-675

19. Heo WI, Lee KE, Hong JY, Kim MN, Oh MS, Kim YS, Kim KW, Kim KE, Sohn MH (2015) The role of interleukin-17 in mouse models of atopic dermatitis and contact dermatitis. Clin Exp Dermatol 40:665-671

20. Ma L, Xue HB, Guan XH, Shu CM, Wang F, Zhang JH, An RZ (2014) The imbalance of Th17 cells and CD4(+) CD25(high) Foxp3(+) Treg cells in patients with atopic dermatitis. J Eur Acad Dermatol Venereol 28:1079-1086

21. Leonardi S, Cuppari C, Manti S, Filippelli M, Parisi GF, Borgia F, Briuglia S, Cannavò P, Salpietro A, Arrigo T et al (2015) Serum interleukin 17, interleukin 23, and interleukin 10 values in children with atopic eczema/dermatitis syndrome (AEDS): association with clinical severity and phenotype. Allergy Asthma Proc 36:74-81

22. Shershakova N, Bashkatova E, Babakhin A, Andreev S, Nikonova A, Shilovsky I, Kamyshnikov O, Buzuk A, Elisyutina O, Fedenko E et al (2015) Allergen-specific immunotherapy with monomeric allergoid in a mouse model of atopic dermatitis. PLoS One 10: $\mathrm{e} 0135070$ 
23. Nakajima S, Kitoh A, Egawa G, Natsuaki Y, Nakamizo S, Moniaga CS, Otsuka A, Honda T, Hanakawa S, Amano W et al (2014) IL$17 \mathrm{~A}$ as an inducer for Th2 immune responses in murine atopic dermatitis models. J Invest Dermatol 134:2122-2130

24. Cedeno-Laurent F, Barthel SR, Opperman MJ, Lee DM, Clark RA, Dimitroff CJ (2010) Development of a nascent galectin-1 chimeric molecule for studying the role of leukocyte galectin-1 ligands and immune disease modulation. J Immunol 185:4659-4672

25. Iqbal AJ, Cooper D, Vugler A, Gittens BR, Moore A, Perretti M (2013) Endogenous galectin-1 exerts tonic inhibition on experimental arthritis. J Immunol 191:171-177

26. Juszczynski P, Ouyang J, Monti S, Rodig SJ, Takeyama K, Abramson J, Chen W, Kutok JL, Rabinovich GA, Shipp MA (2007) The AP1-dependent secretion of galectin-1 by Reed Sternberg cells fosters immune privilege in classical Hodgkin lymphoma. Proc Natl Acad Sci U S A 104:13134-13139

27. Yakushina VD, Vasil'eva OA, Ryazantseva NV, Novitsky VV, Tashireva LA (2015) The effects of galectin-1 on the gene expression of the transcription factors TBX21, GATA-3, FOXP3 and RORC. Mol Cell Biochem 398:245-249

28. Rabinovich GA, Daly G, Dreja H, Tailor H, Riera CM, Hirabayashi J, Chernajovsky Y (1999) Recombinant galectin-1 and its genetic delivery suppress collagen-induced arthritis via $\mathrm{T}$ cell apoptosis. $\mathrm{J}$ Exp Med 190:385-398

29. Santucci L, Fiorucci S, Rubinstein N, Mencarelli A, Palazzetti B, Federici B, Rabinovich GA, Morelli A (2003) Galectin-1 suppresses experimental colitis in mice. Gastroenterology 124:13811394

30. Toscano MA, Commodaro AG, Ilarregui JM, Bianco GA, Liberman A, Serra HM, Hirabayashi J, Rizzo LV, Rabinovich GA (2006) Galectin-1 suppresses autoimmune retinal disease by promoting concomitant Th2- and T regulatory-mediated anti-inflammatory responses. J Immunol 176:6323-6332

31. de la Fuente H, Perez-Gala S, Bonay P, Cruz-Adalia A, Cibrian D, Sanchez-Cuellar S, Dauden E, Fresno M, García-Diez A, SanchezMadrid F (2012) Psoriasis in humans is associated with downregulation of galectins in dendritic cells. J Pathol 228:193-203

32. Yu M, Eckart MR, Morgan AA, Mukai K, Butte AJ, Tsai M, Galli SJ (2011) Identification of an IFN- $\gamma /$ mast cell axis in a mouse model of chronic asthma. J Clin Invest 121:3133-3143

33. Ge XN, Ha SG, Greenberg YG, Rao A, Bastan I, Blidner AG, Rao SP, Rabinovich GA, Sriramarao P (2016) Regulation of eosinophilia and allergic airway inflammation by the glycan-binding protein galectin-1. Proc Natl Acad Sci U S A 113:E4837-E4846

34. Delbrouck C, Doyen I, Belot N, Decaestecker C, Ghanooni R, de Lavareille A, Kaltner H, Choufani G, Danguy A, Vandenhoven G et al (2002) Galectin-1 is overexpressed in nasal polyps under budesonide and inhibits eosinophil migration. Lab Investig 82: $147-158$

35. Vespa GN, Lewis LA, Kozak KR, Moran M, Nguyen JT, Baum LG, Miceli MC (1999) Galectin-1 specifically modulates TCR signals to enhance TCR apoptosis but inhibit IL-2 production and proliferation. J Immunol 162:799-806

36. Barrionuevo P, Beigier-Bompadre M, Ilarregui JM, Toscano MA, Bianco GA, Isturiz MA, Rabinovich GA (2007) A novel function for galectin-1 at the crossroad of innate and adaptive immunity: galectin-1 regulates monocyte/macrophage physiology through a nonapoptotic ERK-dependent pathway. J Immunol 178:436-445

37. Muglia CI, Gobbi RP, Smaldini P, Delgado ML, Candia M, Zanuzzi C, Sambuelli A, Rocca A, Toscano MA, Rabinovich GA et al (2016) Inflammation controls sensitivity of human and mouse intestinal epithelial cells to galectin-1. J Cell Physiol 231:1575-1585 